

$$
\text { JINR E } \begin{aligned}
& \mathrm{E}-93-452 \\
& \text { SW }
\end{aligned}
$$

D.Karadjov ${ }^{1}$, V.V.Voronov, F.Catara ${ }^{2}$

\title{
EFFECT OF GROUND STATE CORRELATIONS \\ ON THE CHARGE TRANSITION DENSITIES \\ OF VIBRATIONAL STATES
}

${ }^{1}$ Institute for Nuclear Research and Nuclear Energy, boul. Tzarigradsko Chaussee 72, 1784 Sofia, Bulgaria

${ }^{2}$ Dipartimento di Fisica dell'Universita' and Instituto Nazionale di Fisica Nucleare, Sezione di Catania, 57, Corso Italia - I 95129 Catania, Italy 


\section{Introduction}

It is well known that many basic features of the nuclear vibrational states can be described within the Random Phase Approximation (RPA), which enables one to treat some correlations in the ground state.

The ground state correlations (GSC) problem has a long story. This problem was discussed in refs. [1-11]. There is a rather complete list of references on that subject in paper[10]. Higher (renormalized) RPA equations, which include corrections for GSC, have been advanced by several authors [1-11]. To apply these methods one needs the oneparticle densities of the correlated ground state. In our calculations we use the approach suggested in $[1,2]$. The generalization of this approach to take into account the effect of GSC on the pairing has been done in ref.[6]. An alternative approach closely related to the one from refs. $[1,2]$ and differing in the way of evaluating the one-particle densities of the correlated state has been proposed in ref.[11].

Being the spatial overlap between the ground state wave function and the excited state wave function the charge transition density provides a good test for nuclear models. The surface nature of the low-lying collective states has been demonstrated in the experiments on inelastic electron scattering from magic nuclei [12]. Such a behavior was predicted by calculations performed within the Hartree-Fock (HF) approach with effective forces [13] and the finite Fermi systems theory [14]. The changes of the nuclear densities due to the zero point fluctuations associated with surface modes in the $\mathrm{Ca}$ isotopes were calculated within the nuclear field theory in ref. $[15]$, where the quasiparticle distribution in the ground state was calculated within the RPA. Recent experimental and theoretical (based on the random phase approximation (RPA)) studies of the charge transition densities $[16,17]$ to investigate the interplay between single-particle and collective degrees of freedom in the excitation of the low-lying states in some spherical nuclei are in reasonable agreement, but the theory gives fluctuations of the transition densities in the interior region. In RPA, as in HF, the theoretical fluctuations are too large in the nuclear interior, which indicates a systematic problem of a more fundamental nature (a detailed discussion can be found in refs. $[18,19])$.

This long standing problem is not solved up to now. In a recent paper [20] we pointed out that an inclusion of the GSC beyond the RPA gives a $30 \%$ depletion of the charge transition densities of the first quadrupole state in the interior region of ${ }^{64} \mathrm{Zn}$. In this case the blocking effect due to the Pauli principle plays an essential role. Nevertheless some discrepancy between the experimental density and the calculated one remains. 
In present paper we extend our approach to take into account the effect of the GSC on the pairing. We consider the charge transition desities of the vibrational states in $\mathrm{Zn}$ isotopes.

\section{Formalism}

We employ a Hamiltonian including an average nuclear field as the Woods-Saxon potential, pairing interactions and isoscalar particle-hole $(p-h)$ residual forces in separable form with the Bohr-Mottelson radial dependence ( see for example $[21,22]$ ).

$$
\begin{aligned}
H & =\sum_{r}\left[\sum_{j m}\left(E_{j}-\lambda_{\tau}\right) a_{j m}^{+} a_{j m}-\frac{G_{\tau}^{(0)}}{4}:\left(P_{0}^{+} \cdot P_{0}\right)^{\tau}:\right. \\
& \left.-\sum_{\lambda \mu} \frac{\kappa^{(\lambda)}}{2}:\left(M_{\lambda \mu}^{+} \cdot M_{\lambda \mu}\right)^{\tau}:\right\rfloor
\end{aligned}
$$

The notation $\{\tau=(n, p)\}$ is used. The single-particle states are specified by the quantum numbers $(\mathrm{jm}) ; E_{j}$ are the single-particle energies; $\lambda_{\tau}$ is the chemical potential; $G_{\tau}^{(0)}$ and $\kappa^{(\lambda)}$ are the strengths in the $\mathrm{p}-\mathrm{p}$ and in the $\mathrm{p}-\mathrm{h}$ channel, respectively. The pair creation and the multipole operators entering the scalar products in (1) are defined in a standard fashion:

$$
\begin{gathered}
P_{0}^{+}=\sum_{j m}(-1)^{j-m} a_{j m}^{+} a_{j-m}^{+} \\
M_{\lambda_{\mu}}^{+}=\frac{1}{\sqrt{2 \lambda+1}} \sum_{j j^{\prime} m m^{\prime}} f_{j j^{\prime}}^{(\lambda)}\left\langle j m j^{\prime} m^{\prime} \mid \lambda \mu\right\rangle a_{j m}^{+} a_{j^{\prime} m^{\prime}}
\end{gathered}
$$

where $f_{j j^{\prime}}^{(\lambda)}$ stands for the reduced single particle matrix element.

By performing the canonical Bogolubov transformation.

$$
a_{j m}^{+}=u_{j} \alpha_{j m}^{+}+(-1)^{j-m} v_{j} \alpha_{j-m}
$$

one can rewrite the Hamiltonian in terms of the bifermion quasiparticle operators and their conjugate ones:

$$
\begin{gathered}
B\left(j j^{\prime} ; \lambda \mu\right)=\sum_{m m^{\prime}}(-1)^{j^{\prime}+m^{\prime}}\left\langle j m j^{\prime} m^{\prime} \mid \lambda \mu\right\rangle \alpha_{j m}^{+} \alpha_{j^{\prime}-m^{\prime}} \\
A^{+}\left(j j^{\prime} ; \lambda \mu\right)=\sum_{m m^{\prime}}\left\langle j m j^{\prime} m^{\prime} \mid \lambda \mu\right\rangle \alpha_{j m}^{+} \alpha_{j^{\prime} m^{\prime}}^{+}
\end{gathered}
$$

We introduce the phonon creation operators

$$
Q_{\lambda \mu i}^{+}=\frac{1}{2} \sum_{j j^{\prime}}\left[\psi_{j j^{\prime}}^{\lambda i} A^{+}\left(j j^{\prime} ; \lambda \mu\right)-(-1)^{\lambda-\mu} \phi_{j j^{\prime}}^{\lambda i} A\left(j j^{\prime} ; \lambda-\mu\right)\right]
$$

where the index $\lambda$ denotes multipolarity and $\mu$ denotes its z-projection in the laboratory system. 
Using the exact commutation relations between fermion operators one can prove that the following relation is valid:

$$
\left\langle 0\left|\left[Q_{\lambda \mu i}, Q_{\lambda^{\prime} \mu^{\prime} i^{\prime}}^{+}\right]\right| 0\right\rangle=\delta_{\lambda \lambda^{\prime}} \delta_{\mu \mu^{\prime}} \sum_{j j^{\prime}}\left(1-q_{j j^{\prime}}\right) \frac{1}{2}\left[\psi_{j j^{\prime}}^{\lambda i} \psi_{j j^{\prime}}^{\lambda i^{\prime}}-\phi_{j j^{\prime}}^{\lambda i} \phi_{j j^{\prime}}^{\lambda i^{\prime}}\right]
$$

where $|0\rangle$ is the ground state (we assume that $\langle 0 \mid 0\rangle=1$ ), $q_{j j^{\prime}}=q_{j}+q_{j^{\prime}}$ and $q_{j}$ presents the quasiparticle distribution in the ground state:

$$
q_{j}=\frac{1}{\sqrt{2 j+1}}\{0|B(j j ; 00)| 0\rangle
$$

One assumes that $|0\rangle$ is the phonon vacuum, i.e.

$$
Q_{\lambda \mu i}|0\rangle=0
$$

and the phonon operators satisfy the linearized equation of motion

$$
\left[H, Q_{\lambda \mu i}^{+}\right]|0\rangle=\omega_{\lambda i} Q_{\lambda \mu i}^{+}|0\rangle
$$

Imposing the condition $q_{j}=0$, one derives the well known RPA equations. Ken-ji Hara did the next step beyond the RPA [1] keeping in eqs. (7),(9),(10) all the terms containing the bifermion operators $B\left(j j^{\prime} ; \lambda \mu\right)$, but replacing the latest by their mean values in the phonon vacuum. In this approach the equations determining the unknowns $q_{j}$ are derived by using eq.(9). As was shown in refs. $[1,20]$, eqs.(7)-(10) result in the following nonlinear system for the phonon energies, amplitudes and quasiparticle distributions in the ground state:

$$
\begin{gathered}
\left(\varepsilon_{j j^{\prime}}-\omega_{\lambda i}\right) \psi_{j j^{\prime}}^{\lambda i}-\frac{\kappa^{(\lambda)} f_{j j^{\prime}}^{(\lambda)} u_{j j^{\prime}}^{(+)}}{2(2 \lambda+1)} \sum_{j_{1} j_{1}^{\prime}}\left(1-q_{j_{1} j_{1}^{\prime}}\right) u_{j_{1} j_{1}^{\prime}}^{(+)} f_{j_{1} j_{1}^{\prime}}^{(\lambda)}\left(\psi_{j_{1} j_{1}^{\prime}}^{\lambda i}+\phi_{j_{1} j_{1}^{\prime}}^{\lambda i}\right)=0 \\
\left(\varepsilon_{j j^{\prime}}+\omega_{\lambda i}\right) \phi_{j j^{\prime}}^{\lambda i}-\frac{\kappa^{(\lambda)} f_{j j^{\prime}}^{(\lambda)} u_{j j^{\prime}}^{(+)}}{2(2 \lambda+1)} \sum_{j_{1} j_{1}^{\prime}}\left(1-q_{j_{1} j_{1}^{\prime}}\right) u_{j_{1} j_{1}^{\prime}}^{(+)} f_{j_{1} j_{1}^{\prime}}^{(\lambda)}\left(\psi_{j_{1} j_{1}^{\prime}}^{\lambda i}+\phi_{j_{1} j_{1}^{\prime}}^{\lambda i}\right)=0 \\
q_{j}=\sum_{\lambda j^{\prime}} \frac{2 \lambda+1}{2 j+1}\left(1-q_{j j^{\prime}}\right)\left(\phi_{j j^{\prime}}^{\lambda i}\right)^{2} \\
\sum_{j j^{\prime}}\left(1-q_{j j^{\prime}}\right)\left[\left(\psi_{j j^{\prime}}^{\lambda i}\right)^{2}-\left(\phi_{j j^{\prime}}^{\lambda i}\right)^{2}\right]-2=0
\end{gathered}
$$

Here $\varepsilon_{j j^{\prime}}$ are the two-quasiparticle energies and $u_{j j^{\prime}}^{(+)}=u_{j} v_{j^{\prime}}+v_{j} u_{j^{\prime}}$. To take into account the effect of GSC on the pairing one needs to modify the standardt BCS equations $[1,6]$. For the gap

$$
\Delta=G_{\tau}^{\prime 1} \sum_{j}(2 j+1) u_{j} v_{j}\left(1-2 q_{j}\right)
$$




$$
\frac{2}{G^{0}}=\sum_{j} \frac{(j+1 / 2)\left(1-2 q_{j}\right)}{\sqrt{\Delta^{2}+\left(E_{j}-\lambda\right)^{2}}}
$$

and from the particle-number condition:

$$
\langle 0|\hat{N}| 0\rangle=N=\sum_{j}(2 j+1)\left[v_{j}^{2}+\left(u_{j}^{2}-v_{j}^{2}\right) q_{j}\right]
$$

it is possible to determine the chemical potential $\lambda$ :

$$
(j+1 / 2)-N=\sum_{j^{\prime}} \frac{\left(j^{\prime}+1 / 2\right)\left(E_{j^{\prime}}-\lambda\right)\left(1-2 q_{j^{\prime}}\right)}{\sqrt{\Delta^{2}+\left(E_{j^{\prime}}-\lambda\right)^{2}}}
$$

The quasiparticle energies $\varepsilon_{j}$ and coefficients $u_{j}, v_{j}$ can be calculated in the same way as within BCS. The factors $\left(1-q_{j j^{\prime}}\right)$ take into account the blocking effect due to the Pauli principle.

As a result we get the self-consistent system of equations to treat the GSC. In the case of $q_{j}=0$ these equations reduce to the usual RPA and BCS equations.

The system of non-linear equations (11)-(18) is solved numerically by means of the auto-regularized Gauss-Newton iteration process $[23,24]$, executed by the program-package REGN [25]. In this way we construct stable solutions of the system with high accuracy.

In the present paper we study the effect of the GSC on the charge transition density for the vibrational states in spherical nuclei. As an example we consider the chain of the even $\mathrm{Zn}$ isotopes with $A=64--70$. The charge transition density is calculated by the formula

$$
\rho_{i}^{\lambda}(r)=\frac{1}{2} \sum_{j j^{\prime}}\left(1-q_{j j^{\prime}}\right) u_{j j^{\prime}}^{(+)}\left(\psi_{j j^{\prime}}^{\lambda_{1}}+\phi_{j j^{\prime}}^{\lambda_{i}}\right) \rho_{j j^{\prime}}^{\lambda}(r)
$$

The expression for the two-quasiparticle charge transition density $\rho_{j j^{\prime}}^{\lambda}(r)$ can be found for example in [16]. The calculated charge transition densities $\rho_{i}^{\lambda}(r)$ are folded with the formfactor of the proton charge distribution [26]. Knowing the charge transition density it is possible to calculate the reduced transition probabilities [27].

\section{Details of calculations and results}

We performed numerical calculations for the $\mathrm{Zn}$ isotopes for which the experimental data [28] and the RPA results [29] are known. The Woods-Saxon potential parameters in use are basically the same as in our previous paper $[20]$. They were chosen to reach a reasonable theoretical description of the experimental ground state density and the r.m.s. radius [30]. The pairing constants $G_{\tau}^{0}$ are fixed so as to reproduce the odd-even mass difference of neighboring nuclei. We always adjust the strength parameters $\kappa^{(\lambda)}$ so that the $B(E \lambda)$ values were close to the experimental ones. 
The system of eqs. (11)-(18), which treats the GSC self-consistently, describes (via eq.(13)) the coupling between different vibrations and between all the phonon roots of a certain multipolarity. Our studies [20] show that the interplay of different roots of the system of eqs.(11)-(14) is not essential: the contribution from the second root for example affects the $q_{j}$ by no more than $2 \%$. Thus, one can restrict the sum in eq. (13) to only the first (collective) root without substantial loss of accuracy.

To study the coupling between different vibrations we took into account $\lambda=2,3,4$ terms in eqs. (11)-(13), using the same values for the quadrupole, octupole and hexadecapole constants. According to our calculations for ${ }^{64} \mathrm{Zn}$ the most essential role for the GSC is played by the quadrupole and octupole vibrations. The values of $q_{j}$ calculated for the pure quadrupole vibrations(i.e. $\lambda=2$, only) are as a rule a few times higher than those for the pure octupole vibrations $(\lambda=3$, only). However, in the case of coupled $(\lambda=2,3)$ vibrations the resulting $q_{j}$ are larger than the sum of $q_{j}(\lambda=2)$ and $q_{j}$ $(\lambda=3)$ contrary to the RPA case because of the non-linear character of the $\lambda$-mixing. The admixture of the hexadecapole vibrations changes $q_{j}$ by no more than $5 \%$. So in what follows we take into account the mixing of the quadrupole and octupole modes only.

The results of our calculations for the quasiparticle distribution in the ground state of ${ }^{66} Z n$ are shown in the table 1 . The table 1 contains the results when we take into account the effect of GSC on $q_{j}$ but neglect the blocking in the pairing due to GSC $\left(q_{j}(\right.$ Hara $)$ ) and when the blocking is included $\left(q_{j}(B-H a r a)\right)$. The $q_{j}$ calculated within the RPA [3] ass

$$
q_{j}=\frac{1}{2} \sum_{\lambda i j^{\prime}} \frac{2 \lambda+1}{2 j+1}\left(\phi_{j j^{\prime}}^{\lambda i}\right)^{2}
$$

are given in the same table too. As one can see from the table 1 the $q_{j}$ have large values for the subshells near the Fermi surface only and the Hara approach gives stronger correlations in comparison with the RPA. The inclusion of the blocking in the pairing due to GSC results in an additional enhancement of correlations. A similar behaviour of $q_{j}$ 's was found for all $\mathrm{Zn}$ isotopes.

The theoretical and experimental values [28-29] for energies and the reduced transition probabilities of the first quadrupole and octupole states in the $\mathrm{Zn}$ isotopes are shown in tables 2,3. As one can see from the table 2 the energy of the $2_{1}^{+}$state calculated within the RPA with the above choice of parameters is usally higher than the experimental value. In the same time the solution of the GSC problem beyond the RPA exhibits a shift up of the energy $\omega_{2+}$ and an essential reduction of the $B(E 2)$-value. To restore the $B(E 2)$ within the Hara approach we had to increase, for example, the value of $\kappa^{(2)}$ by $38 \%$ and $23 \%$ in ${ }^{64} Z n$ and ${ }^{68} Z n$, respectively. In the last case the resulting energies are shifted down a little. The selfconsistent calculations taking into account the blocking in the pairing give 
an additional shift down in energies, which are in a better agreement with experimental data. The increase of $\kappa^{(2)}$ is about $12 \%$ for all isotopes in comparison with the RPA values.

It is worth mentioning that the value of the $\kappa^{(2)}$ for the non-linear problem is quite larger than the critical RPA constant where the RPA solution becomes complex. For such values of $\kappa^{(2)}$ the use of the procedure [7] where one solves first eqs. (11)-(12) with $q_{j}=0$ and then eq.(13) becomes non-applicable.

It is seen from the table 3 that the GSC with the coupled vibrations change drastically the $3_{1}^{-}$energies. The renormalized values of $\kappa^{(3)}$ exceed the RPA ones by $11-19 \%$ and they are smaller than the critical values, in contrast to the quadrupole constants. In the cases of ${ }^{66} Z n$ and ${ }^{68} Z n$, where the experimental $\mathrm{B}(\mathrm{E} 3)$-values are known, our choice of parameters enables us to reproduce energies and transition probabilities simultaneously. We overesimate apparently the collectivity of the $3_{1}^{-}$state in ${ }^{70} \mathrm{Zn}$.

It should be noted also that according to our calculations the selfconsistent inclusion of the GSC in the pairing problem (see eqs. (15)-(18)) garantees the number conservation with a high accuracy. Calculations without taking into account the GSC effect on the pairing lead sometimes up to a $3 \%$ difference from the exact particle number.

The figures 1-4 show the transition charge densities from the ground to the first $2^{+}$ states in the $\mathrm{Zn}$ isotopes. Our calculations for the $\rho_{i}^{\lambda}(r)$ give results which are similar to the ones of [29], obtained in the RPA with the Skyrme forces, but in contrast with [29] we did not assign occupation probability to each single-particle orbital empirically. As it was pointed in [29] the authors were enforced to destroy the self-consistency shifting the single-particle spectrum for the unoccupied orbitals with respect to the occupied ones to reproduce the experimental value for the first $2^{+}$state.

As one can see from the figures 1-4 the RPA reproduces the behaviour of the charge transition densities qualitatively but it overestimates the interior part of the $\rho_{i}^{\lambda}(r)$. The inclusion of the GSC beyond the RPA (the Hara approach) gives a 30\%-35\% depletion of the maximum of the $\rho_{i}^{\lambda}(r)$ in the interior region of the nuclei. The selfconsistent treatment of the GSC results in a suppression of interior oscilations by factors 2.3 (for ${ }^{68} Z n$ ) -3.4 (for ${ }^{70} Z n$ ). The calculated $\rho_{i}^{\lambda}(r)$ for ${ }^{64} Z n$ and ${ }^{66} Z n$ are very close to the experimental data. Such a depletion is related with the Pauli blocking effect for the proton two-quasiparticle configuration $\left\{2 p_{3 / 2}, 2 p_{3 / 2}\right\}$, which is mainly responsible for the interior bump in the charge transition densities in the $\mathrm{Zn}$ isotopes. According to our RPA calculations the proton twoquasiparticle configuration $\left\{2 p_{3 / 2}, 2 p_{3 / 2}\right\}$ gives a contribution about $38 \%$ and about $46 \%$ into the norm of the first quadrupole phonon in ${ }^{66} \mathrm{Zn}$ and in ${ }^{68} \mathrm{Zn}$, respectively. The 
inclusion of the GSC redistributes the strength of this configuration over many phonon roots and as result the contribution into the first root becomes $8.8 \%$ in ${ }^{66} \mathrm{Zn}$ and $15.6 \%$ in ${ }^{68} \mathrm{Zn}$. As it follows from the eq. (19) the GSC supresses the contribution of the partial two-quasiparticle transition densities having big $q_{j j^{\prime}}$. It is seen from the table 1 that the $q_{2 p_{3 / 2}}$ is the biggest for protons and as it was mentioned above plays an essential role in the structure of the interior part of the transition density for the $2_{1}^{+}$states. The configuration $\left\{1 f_{5 / 2}, 1 f_{5 / 2}\right\}$ gives some contribution in the interior part too and the same mechanism of a supression takes place for it. The amplitudes of the oscilations for the configurations with low orbital momenta are bigger than for the ones with high orbital momenta.

We would like to emphasize that this effect can not be reproduced by any renormalization of the $\kappa^{(2)}$ in the RPA.

The behaviour of the neutron transition densities differs from the proton ones (see figs.5,6). In the case of ${ }^{66} Z n$, for example, the influence of the GSC on the interior part of the transition density is very weak because the neutron configuration $\left\{2 p_{3 / 2}, 2 p_{3 / 2}\right\}$ contributes not more than $1.5 \%$ in the norm of the $2_{1}^{+}$state, and the contribution of the configuration $\left\{1 f_{5 / 2}, 1 f_{5 / 2}\right\}$ remains practically the same (about $8 \%$ ) in all cases. An extra four neutrons in ${ }^{70} Z n$ changes the structure of the neutron part of the $2_{1}^{+}$wave function and the GSC affects mainly the surface part of the neutron transition density. In the this case the configurations which are responsible of the interior oscillations in ${ }^{66} Z n$ have very small contribution to the structure of the first quadrupole state.

We calculated the charge transition density for the one-phonon $4^{+}$state and did not find any essential oscillations in the interior region. That is due to the lack of the configuration $\left\{2 p_{3 / 2}, 2 p_{3 / 2}\right\}$ in such states because of the angular momentum coupling rule.

The fig. 7 presents the charge transition density for the $3_{1}^{-}$state in ${ }^{66} Z n$. This density has a clear surface nature and there are no strong oscillations in the interior region of the nucleus because of a destructive intenference of the two-quasiparticle partial transition densities constructed from the single-particle wave functions with different parity. The same picture takes place in the other $\mathrm{Zn}$ isotopes and it is typical for the transition densities of the octupole vibration states (see [16-19]).

It is interesting to note that the GSC increase slightly the ground state r.m.s. charge radius up to $4.05 \mathrm{fm}$ in ${ }^{66} \mathrm{Zn}$. Its $\mathrm{RPA}$ value is $4.02 \mathrm{fm}$, compared to the experimental one $3.99 \mathrm{fm}[30]$. 
Table 1. The quasiparticle distribution in the ground state of ${ }^{66} \mathrm{Zn}$

\begin{tabular}{|c|c|c|c|c|c|c|}
\hline \multirow[t]{2}{*}{$\mathrm{nlj}$} & \multicolumn{3}{|c|}{ neutrons } & \multicolumn{3}{|c|}{ protons } \\
\hline & $q_{j}(\mathrm{RPA})$ & $q_{j}($ Hara $)$ & $q_{j}(\mathrm{~B}$-Hara $)$ & $q_{j}(\mathrm{RPA})$ & $q_{j}($ Нага $)$ & $q_{j}(\mathrm{~B}$-Hara $)$ \\
\hline $2 s_{1 / 2}$ & 0.0052 & 0.0170 & 0.0249 & 0.0132 & 0.0444 & 0.0674 \\
\hline $1 f_{7 / 2}$ & 0.0155 & 0.0492 & 0.0723 & 0.0307 & 0.0907 & 0.1394 \\
\hline $2 p_{3 / 2}$ & 0.0561 & 0.1640 & 0.2286 & 0.0894 & 0.2195 & 0.2425 \\
\hline $1 f_{5 / 2}$ & 0.0438 & 0.1180 & 0.1688 & 0.0333 & 0.0959 & 0.1039 \\
\hline $2 p_{1 / 2}$ & 0.0722 & 0.1791 & 0.2476 & 0.0367 & 0.0960 & 0.0930 \\
\hline $1 g_{9 / 2}$ & 0.0204 & 0.0685 & 0.0997 & 0.0164 & 0.0564 & 0.0701 \\
\hline
\end{tabular}

Table 2. Energies and transition probabilities for the $2_{1}^{+}$states in the $\mathrm{Zn}$ isotopes

\begin{tabular}{|c|cc|cccc|}
\hline A & \multicolumn{2}{|c|}{ Experiment } & \multicolumn{4}{|c|}{ Calculation } \\
\hline & $\omega, M e V$ & $B(E 2 \uparrow), e^{2} f^{4}$ & $\omega_{R P A}, M e V$ & $\omega_{H}, M e V$ & $\omega_{B-H}, M e V$ & $B(E 2 \uparrow), e^{2} f m$ \\
\hline 64 & 0.992 & 1597 & 1.16 & 1.09 & 0.70 & 1597 \\
66 & 1.039 & 1426 & 1.33 & 1.27 & 1.02 & 1428 \\
68 & 1.077 & 1360 & 1.39 & 1.33 & 1.25 & 1360 \\
70 & 0.885 & 2050 & 1.32 & 1.25 & 1.05 & 2050 \\
\hline
\end{tabular}


Table 3. Energies and transition probabilities for the $3_{1}^{-}$states in the $\mathrm{Zn}$ isotopes

\begin{tabular}{|c|cc|cccc|}
\hline A & \multicolumn{2}{|c|}{ Experiment } & \multicolumn{4}{|c|}{ Calculation } \\
\hline & $\omega, M e V$ & $B(E 3 \uparrow), e^{2} f m^{6}$ & $\omega_{R P A}, M e V$ & $\omega_{H}, M e V$ & $\omega_{B-H}, M e V$ & $B(E 3 \uparrow), e^{2} f m^{0}$ \\
\hline 64 & 2.999 & - & 3.90 & 3.03 & 2.69 & 44000 \\
66 & 2.830 & 41660 & 3.89 & 3.19 & 2.83 & 41660 \\
68 & 2.751 & 38460 & 3.62 & 3.06 & 2.75 & 38460 \\
70 & 2.839 & - & 3.55 & 2.77 & 2.32 & 36000 \\
\hline
\end{tabular}

\section{Conclusion}

In conclusion, we point out that the system of non-linear equations, describing the ground state correlations beyond RPA selfconsistently was solved in a realistic case. It was found that such a proper treatment of the GSC leads to a suppression by factors 2.3-3.4 in comparison to the RPA of the charge transition density in the interior region of the $\mathrm{Zn}$ isotopes. It follows from our study that the inclusion of the blocking effect due the Pauli prinsiple enables us to solve the long standing problem of the theoretical overatimation of the charge transition densities in the interior region of nuclei for the positive parity vibration states compared to experimental data.

Our preliminary investigation shows that taking into account the blocking effect for the proton configuration $\left\{2 d_{5 / 2}, 2 d_{5 / 2}\right\}$ it is possible to get a good description of the interior patr of the charge transition densities of $2^{+}, 4^{+}$states in the $\mathrm{Nd}$ isotopes. To describe the surface part one has to take into account in addition the interplay between the onephonon and more complex configurations, so the system of non-linear equations should be extended. Such calculations are in progress now. 


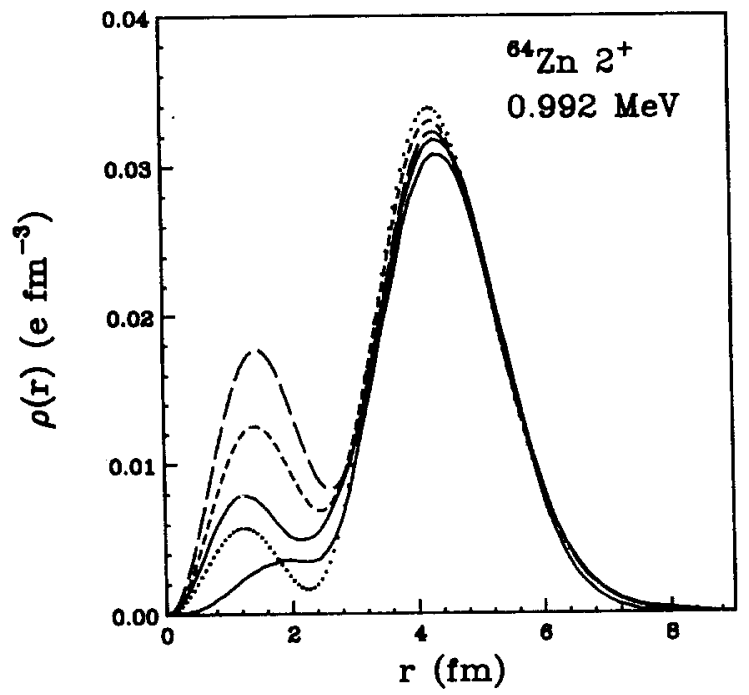

Fig.1: Transition charge density from the ground state to the first $2_{1}^{+}$ state in ${ }^{6+} Z n$.

solid curves - experimental data [28]; long dashed curve - RPA calculation; dashed curve - results with GSC beyond the RPA without the blocking in the pairing; dotted curve - results with the selfconsistent inclusion of GSC beyond the RPA.

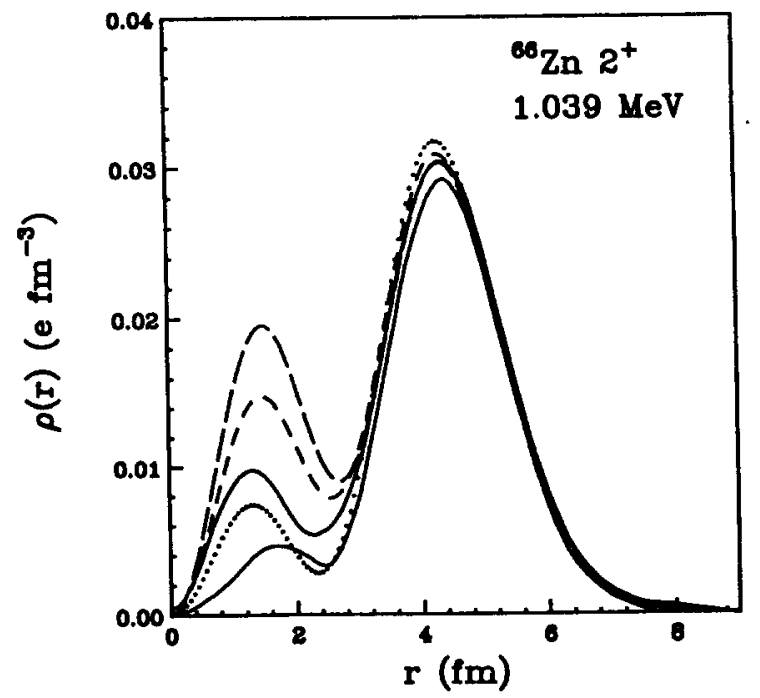

Fig.2: Transition charge density from the ground state to the first $2_{1}^{+}$ state in ${ }^{66} \mathrm{Zn}$.

Notations are the same as in fig.1. 


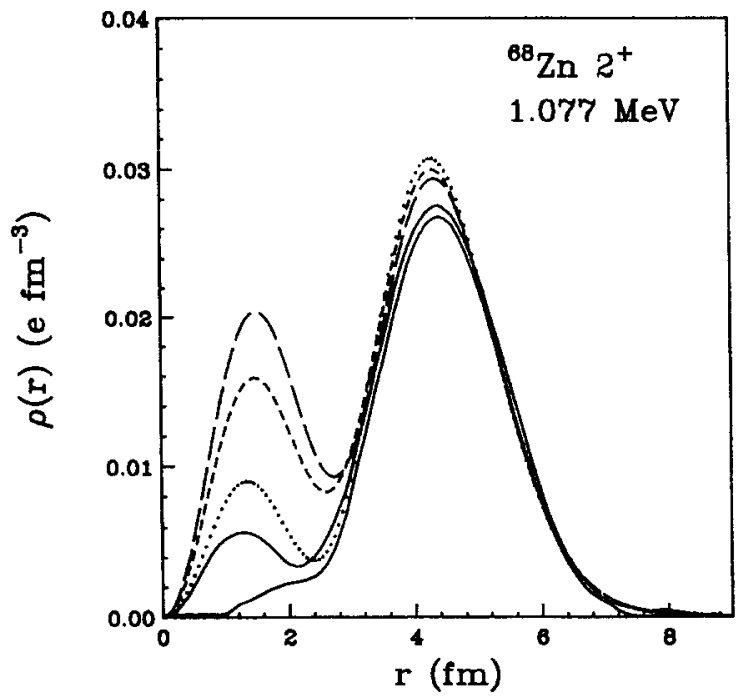

Fig.3: Transition charge density from the ground state to the first $2_{1}^{+}$ state in ${ }^{68} \mathrm{Zn}$.

Notations are the same as in fig.1.

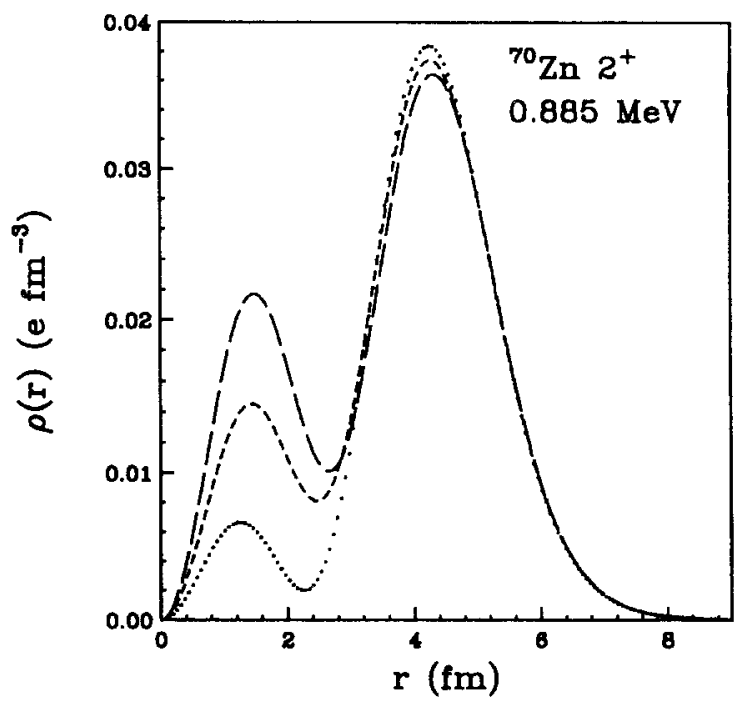

Fig.4: Transition charge density from the ground state to the first $2_{1}^{+}$ state in ${ }^{i n} \mathrm{Z} n$.

Notations are the same as in fig.1. 


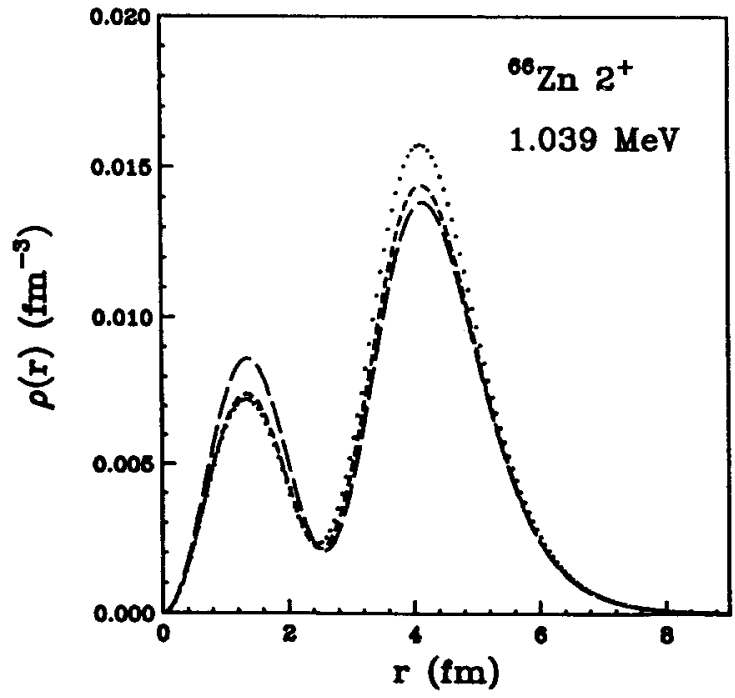

Fig.5: Neutron transition density from the ground state to the first $2_{1}^{+}$ state in ${ }^{66} \mathrm{Zn}$.

long dashed curve - RPA calculation;

dashed curve - results with GSC beyond the RPA without the blocking in the pairing; dotted curve - results with the selfconsistent inclusion of GSC beyond the RPA.

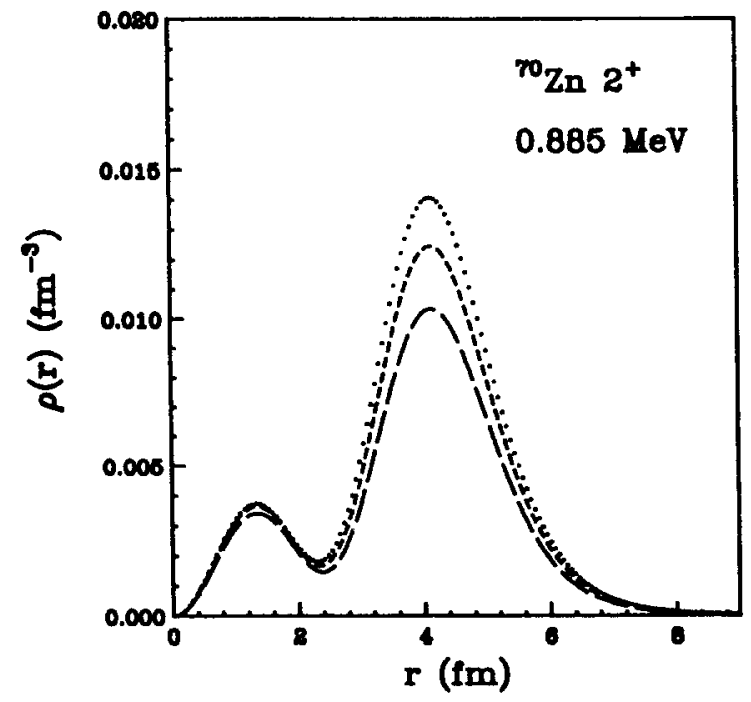

Fig.6: Neutron transition density from the ground state to the first $2_{1}^{+}$ state in ${ }^{70} \mathrm{Zn}$.

Notations are the same as in fig.5. 




Fig.7: Transition charge density from the ground state to the first 3 , state in ${ }^{66} Z n$.

Notations are the same as in fig.5.

This work has been partially supported by the Bulgarian Nation Science Foundation under contract $\Phi-310$. Two of us (D.K. and V.V.V.) thank the INFN for the hospitality and support during their stay in Catania, where a part of this work was done.

\section{References}

[1] Ken-ji Hara 1964 Prog. Theor. Phys. 3288.

[2] Ikeda K Udagawa T and Yamaura H. 1965 Prog. Theor. Phys. 3322.

[3] Rowe D J 1968 Phys. Rev. 1751283.

[4] Rowe D J 1968 Rev. Mod. Phys. 40153.

[5] J. da Providencia 1968 Nucl. Phys. A108589.

[6] Jolos R V and Rybarska W 1980 Z. Physik A 29673.

[7] Navrotska-Rybarska W Stoyanova O and Stoyanov Ch 1980 Yad. Fiz. 331494.

[8] Johnson C H and Mahaux C 1988 Phys. Rev. C38 2589.

[9] Lenske H and Wambach J 1990 Phys. Lett. B249 377. 
[10] Klein A Walet N R and Do Dang G 1991 Nucl. Phys. A535 1.

[11] Catara F Nguyen Dinh Dang and Sambataro M 1993 Submitted to Nucl. Phys. A.

[12] Heisenberg J et al. 1984 Phys. Rev. C25 2292.

[13] Bertsch G F and Tsai S F 1975 Phys. Rep. C18 125.

[14] Fayans S A Khodel V A and Saperstein E E 1979 Nucl. Phys. A317 424.

[15] Broglia R A Barranco F and Gallardo M 1985 "Nuclear Structure 1985" ed by Broglia R A Hagemann G B and Herskind B, Elsevier Science Publishers B.V. 193.

[16] Sandor R K J et al 1991 Nucl. Phys. A535 669.

[17] Kim W et al 1991 Phys. Rev. C44 2400.

[18] Heisenberg J and Block H P 1983 Ann. Rev. Nucl. Part. Sci. 33569.

[19] Frois B and Papanicolas C N 1987 Rev. Nucl. and Part. Sci. 37133.

[20] Karadjov D Voronov V V and Catara F 1993 Phys. Lett. B306 197.

[21] Bohr A and Mottelson B R 1975 "Nuclear Structure" vol.2 Benjamin New York.

[22] Soloviev V G 1987 Prog. in Part. and Nucl. Phys.19 107.

[23] Aleksandrov L J. 1971 Comput. Mat. and Mat. Fiz. 11 36;

[24] Aleksandrov L 1973 Preprints JINR P5-7258,P5-7259 Dubna.

[25] Aleksandrov L Drenska M and Karadjov D 1981 "Program System REGN for Solution of Nonlinear Systems of Equations" PSR-165/ REGN RSIC Oak Ridge Ten. nessee.

[26] Simon G G et al 1980 Nucl.Phys. A333 381.

[27] Satchler G R 1983 "Direct Nuclear Reactions" New York-Oxford Univ. Press.

[28] Neuhausen R 1977 Nucl.Phys. A282 125.

[29] Tsai S F and Bertsch G F 1975 Phys.Lett. B59 425.

[30] De Vries H et al 1987 Atomic Data and Nucl. Data. Tables 36495. 
Принимается подписка на препринты, сообщения Объединенного института ядерных исследований и «Краткие сообщения ОИЯИ».

Установлена следующая стоимость подписки на 12 месяцев на издания ОИЯИ, включая пересылку, по отдельным тематическим категориям:

\begin{tabular}{lr}
\hline Индекс & $\begin{array}{r}\text { Цена подпискик } \\
\text { на год }\end{array}$ \\
\hline 1. Экспериментальная физика высоких энергий & $915 \mathrm{p}$. \\
\hline 2. Теоретическая физика высоких энергий & $2470 \mathrm{p}$. \\
\hline 3. Экспериментальная нейтронная физика & $365 \mathrm{p}$. \\
\hline 4. Теоретическая физика низких энергий & $735 \mathrm{p}$. \\
\hline 5. Математика & $460 \mathrm{p}$. \\
\hline 6. Ядерная спектроскопия и радиохимия & $275 \mathrm{p}$. \\
\hline 7. Физика тяжелых ионов & $185 \mathrm{p}$. \\
\hline 8. Криогеника & $185 \mathrm{p}$. \\
\hline 9. Ускорители & $460 \mathrm{p}$. \\
\hline 10. Автоматизация обработки экспериментальных данных & $560 \mathrm{p}$. \\
\hline 11. Вычислительная математика и техника & $560 \mathrm{p}$. \\
\hline 12. Химия & $90 \mathrm{p}$. \\
\hline 13. Техника физического эксперимента & $720 \mathrm{p}$. \\
\hline 14. Исследования твердых тел и жидкостей ядерными методами & $460 \mathrm{p}$. \\
\hline
\end{tabular}

15. Экспериментальная физика ядерных реакций при низких энергиях 460 p.

16. Дозиметрия и физика защиты $90 \mathrm{p}$.

17. Теория конденсированного состояния $365 \mathrm{p}$.

\section{8. Использование результатов}

и методов фундаментальных физических исследований

в смежных областях науки и техники

$90 \mathrm{p}$.

19. Биофизика $185 \mathrm{p}$.

«Краткие сообщения ОИЯИ» (6 выпусков) $560 \mathrm{p}$.

Подписка может быть оформлена с любого месяца года.

По всем вопросам оформления подписки следует обращаться в издательсктий отдел ОИЯИ по адресу: 141980, г.Дубна, Московской области 


\section{SUBJECT CATEGORIES \\ OF THE JINR PUBLICATIONS}

Index

\section{Subject}

1. High energy experimental physics

2. High energy theoretical physics

3. Low energy experimental physics

4. Low energy theoretical physics

5. Mathematics

6. Nuclear spectroscopy and radiochemistry

7. Heavy ion physics

8. Cryogenics

9. Accelerators

10. Automatization of data processing

11. Computing mathematics and technique

12. Chemistry

13. Experimental techniques and methods

14. Solid state physics. Liquids

15. Experimental physics of nuclear reactions at low energies

16. Health physics. Shieldings

17. Theory of condensed matter

18. Applied researches

19. Biophysics 
Караджов Д., Воронов В.В., Катара Ф.

Влияние корреляций в основных состояниях

на зарядовые переходные плотности вибрационных состояний

Изучено влияние корреляций в основных состояниях на зарядовые переходные плотности вибрационных состояний в сферических ядрах. Выход за приближение случайных фаз приводит к необходимости решать нелинейную систему уравнений. При этом также учитывается влияние корреляций на спаривание. Показано, что учет корреляций в основных состояниях приводит к существенному подавлению зарядовых переходных плотностей во внутренней области ядер по сравнению с расчетами в приближении случайных фаз и позволяет описать экспериментальные данные.

Работа выполнена в Лаборатории теоретической физики им. Н.Н.Боголюбова ОИЯИ.

Сообщение Объединенного института ядерных исследований. Дубна, 1993

Karadjov D., Voronov V.V., Catara F.

Effect of Ground State Correlations

E4-93-452

on the Charge Transition Densities of Vibrational States

The effect of ground state correlations on the charge transition densities of vibrational states in spherical nuclei is studied. The problem for the ground state correlations beyond RPA leads to a non-linear system of equations, which is solved numerically. The influence of the correlations on the pairing is taken into account too. The inclusion of ground state correlations beyond RPA results in an essential suppression of the charge transition density in the nuclear interior in comparison with the RPA calculations and enables one to reproduce the experimental data.

The investigation has been performed at the Bogoliubov Laboratory of Theoretical Physics, JINR. 
$16 \mathrm{p}$.

\author{
Макет Т.Е.Попеко \\ Подписано в печать 24.01.94 \\ Формат 60×90/16. Офсетная печать. Уч.-изд. листов 1,38 . \\ Тираж 370. Заказ 46937.
}

Издательский отдел Объединенного института ядерных исследований Дубна Московской области 